Open Access

\title{
Correction to: A supplemental device to return escaping particles to a magnetic mirror reactor
}

Mitsuaki Nagata $^{{ }^{*}}$ and Keiichi Sawada ${ }^{2}$

\footnotetext{
*Correspondence: scc.kei@gmail. com

${ }^{1}$ Nippon Electronic Engineering College, Noboribetsu-shi, Hokkaido, Japan

Full list of author information is available at the end of the article
}

\section{Correction}

In the publication of this article [1], contained the following errors. In this Correction, the affected parts of the article are shown. The list of references is available in the original article. We omit pointing out obvious errata with respect to unnecessary periods and wrong symbols.

$$
\begin{aligned}
& \text { "In Eq.(2) }\left\{\frac{m_{e}}{\left(1-\frac{v_{0}^{2}}{c^{2}}\right)^{2}}+\int_{t_{0}}^{t} \frac{-q \mathrm{E}(t) \cdot \cdot\left(t, t_{0}\right)}{c^{2}} d t\right\} \frac{\partial v\left(t, t_{0}\right)}{\partial t}+\boldsymbol{v}\left(\boldsymbol{t}, \boldsymbol{t}_{\mathbf{0}}\right)\left(\frac{-q \mathrm{E}(t) \cdot \boldsymbol{v}\left(\boldsymbol{t}, \boldsymbol{t}_{0}\right)}{c^{2}}\right)=-q \mathbf{E}(t)-q v\left(\boldsymbol{t}, \boldsymbol{t}_{\mathbf{0}}\right) \times \mathbf{B} \\
& \text { "In Eq.(6) } \frac{m_{e}}{\sqrt{1-\frac{v_{0}^{2}}{c^{2}}}} \equiv m, \frac{q B}{m_{e}} \sqrt{1-\left(\frac{v_{0}^{2}}{c^{2}}\right)}=\frac{q B}{m} \equiv \omega_{c},\left(c^{\prime \prime}=c^{2}\right) \\
& \text { "In Eq.(9), } c\left(z_{0} C\right) \\
& \hat{z}\left(v_{x}^{\prime} B\right) \\
& \text { "In Eq.(15) } \frac{q E}{m} \frac{-\omega_{c}}{\omega_{c}^{2}-\omega^{2}} \\
& \text { "In Eq.(17) }-\frac{1}{4} \frac{q E}{m \omega_{c}} \frac{z_{0}^{2}}{c}\left(\omega_{c}^{2} t^{2}+3 S^{2}\right) C \\
& \text { "In Eq.(21) } \frac{-\omega_{c}}{\omega_{c}^{2}-\omega^{2}}\left(\frac{-1}{4}\right) \\
& \frac{\sin \left(2 \omega_{c}-\omega\right) t}{2 \omega_{c}-\omega}-\frac{2 s i n(\omega t}{\omega} \\
& \text { "In Fig.9; n=1670 } \\
& \text { " Reference (14): J Plasma Phys. 44:47 }
\end{aligned}
$$

This has now been included in this correction.

\section{Author details \\ ${ }^{1}$ Nippon Electronic Engineering College, Noboribetsu-shi, Hokkaido, Japan. ${ }^{2}$ Soft Creator Company, Shinmachi, Nakagyo-ku, Kyoto, Japan.}

\section{Received: 12 February 2018 Accepted: 8 May 2018}

Published online: 03 September 2018

\section{Reference}

1. Nagata, Sawada (2018) A supplemental device to return escaping particles to a magnetic mirror reactor. 5:1 https://doi.org/10.1140/epjti/s40485-018-0042-4 